\title{
Ueber die Languriiden-Arten von Kamerun nebst einigen verwandten Formen.
}

$$
\text { Von }
$$

\section{Dr. G. K r a at z.}

Fowler hat in den Transactions der Londoner entomologischen Gesellschaft fünf Arten von Kamerun beschrieben, welche von mir in dieser Zeitschrift 1895 S. 201 unter den africanischen Arten aufgezählt sind.

Unter den Arten, welche Conrad auf Johann Albrechts-Höhe (Nord-Kamerun) und in Lolodorf gesammelt hat, fand sich nur 1 Ex. der Gattung Stenolanguria und keine einzige Art der Gattung Promecolanguria ${ }^{1}$ ) Fowler, auf welche ich fünf von Conrad bei Togo gesammelte Languria beziehen zu können glaıbe; Languria gracilior und acuminipennis Krtz. stehen der Promecolanguria zunächst, dagegen ist die neue Gattung Ischnolanguria (mit 2 Arten) auf Albrechts-Höhe angewiesen und durch ihre eigenthümliche schlanke Gestalt ausgezeichnet. Languria spectabilis, dubia Fowl., Murrayi Fowl., dolens Krtz. und semirufa Krtz. scheinen echte Langurien zu sein; über die kleine Languria macilenta Krtz. wage ich nach einem Ex. kein bestimmtes Urtbeil zu fällen; auf castanea Krtz. stellte ich die Gattung Platycladoxena auf. Penolanguria nigerrima Krtz. ist sicher eine Penolanguria, ob aber wirklich eine Languriide? da sie durch die Stellung des Kopfes von allen bekannten Arten abweicht.

Soweit über die besprochenen Arten im Allgemeinen.

1) Ob Languria calcaroides Quedenf. (Berl. Ent. Ztschr. 1888, p. 207) wirklich von Nyassae Fowler (Trans. Ent. Soc. London 1885, p. 384) verschieden ist, scheint mir insofern zweifelhaft, als rothe Fühler leicht durch Feuchtigkeit schwärzlich-braun erscheinen können und Fowler das deutlich zweibuchtige Halssch. vielleicht nicht bemerkt haben kann; ob Quedenfeldt wirklich eine kürzere Art vom See Nyassa vor sich gehabt hat, ist aus dem Texte nicht ersichtlich.

Fowler schlägt für seine Nyassae den Namen Promecolanguria vor, der wohl jedenfalls für calcaroides einzutreten hat; ich vermuthe, dals Languria dimidiata Krtz., ruficollis Krtz., brunnea Krtz., rufobrunnea Krtz. und semirufa Krtz. (Deutsche Ent. Zeitschr. 1895, p. 202-204) ebenfalls zu Promecolanguria gehören. 
Ischnolanguria.

Statura gracilis.

Antennarum clava 5-articulata, scapo longitudine fere aequali. Abdominis segmentum primum lineis ventralibus duabus.

Elytra punctalo-striata, posterius leviter altenuala, apice rolundala.

Zwei habituell sehr ähnliche, schlanke, ziemlich grofse Arten scheinen mir ihrer Fühlerbildung halber von den übrigen Langurien abgesondert werden zu können; die fünfgliedrige Keule, auf welche die Gattung Pentelanguria (die vorzugsweise in Indien und im malayischen Archipel zu Hause ist) begründet wurde, hat bei Pentelanguria etwa nur ein Drittheil der Länge des ganzen Fühlers, während sie bei Ischnolanguria fast so lang wie der übrige Theil desselbelben ist; das sechste Glied ist hier sogar bei einzelnen Männchen deutlich nach aulsen erweitert. Die Vordertarsen der Männchen sind merklich stärker erweitert als die der Weibchen.

1. Ischnolanguria concolor: Elongata, tota cyanea, antennarum clava lata, 5-articulata, articulis tertio maris latitudine paullo longiore, feminae secundo subaequali, thorace elongato, apicem versus leviter attenuato, leviter convexo, crebre punctato, basi haud striolato, elytris thorace paullo latioribus, apicem versus leviter angustatis, apice rotundatis, subtiliter punclato-striatis. - Long. $10-11$ lin.

An der schlanken Gestalt und der grofsen fünfgliedrigen Fühlerkeule leicht zu erkennen, einfarbig schön bläulich. Languria calabarensis Fowler und dubia Fowler (siehe die Citate in der Deutschen Entomol. Zeitschr. 1895, p. 201) haben eine breite viergliedrige Keule. Der Kopf ist mälsig dicht fein punktirt. Das Halssch. ist fast um die Hälfte länger als breit, vorn leicht verschmälert, ziemlich gewölbt, mäfsig dicht, fein punktirt, an der Basis leicht der Quere nach vertieft, o hne Längsstriche. Das Schildchen ist meist ziemlich stark vertieft. Die Fld. sind an der Basis etwas breiter als der Thorax, nach hinten leicht verschmälert, an der Spitze aufsen sehr deutlich abgerundet, innen weniger deutlich, der Aufsenrand etwas aufgebogen; die Punktstreifen sind fein und regelmälsig, fast bis zur Spitze reichend. Brust und Hinterleib sind hinten in der Mitte glatt, an den Seiten weitläufig punktirt.

2. Ischnolanguria Conradti: Elongata, cyanea, pectore, abdomine pedibusque (geniculis exceptis) rufo-testaceis, antennarum clava lata, 5-articulata. - Long. 9-10 $\frac{1}{2}$ mill. 
Der vorhergehenden sehr ähnlich, aber durch die lebhaft gelbrothe Färbung der Brust, des Hinterleibes und der Füfse leicht zu unterscheiden; andere gute Unterschiede habe ich kaum aufzufinden vermocht und würde den Käfer für eine Farben-Varietät des vorhergehenden halten, wenn Uebergänge und Analogien vorhanden wären.

Nicht ganz so selten wie der vorhergehende.

3. Languria dubia Fowler (Trans. Ent. Soc. London 1885, p. 385). Einige Ex. von Kamerun, darunter einige Ex. ohne rothe Wurzel der Schenkel (var. nigripes Kr. etc.).

4. Languria Murrayi Fowler (Trans. Ent. Soc. London 1885 , p. 386). Einige Ex. von Johann Albrechts-Höhe glaube ich auf diese Art beziehen zu können.

5. Languria dolens: Elongata, tota nigro-cyanea, capite triangulari, vix punctulato, oculis prominentibus, antennis minus robustis, clava triarticulata (vix quadriarticulata), thorace subquadrato, convexiusculo, parce punctulato, basi impresso, utrinque striolato, elytris thoracis medio fere latioribus, apicem versus parum coarctatis, seriatim subtiliter punctatis, utroque subacuminato, pedibus concoloribus, tarsis leviter dilatatis, abdomine parce punctato, basi striolato. - Long. 6 mill.

Von der dunklen Färbung der vorhergehenden Arten, aber viel kleiner, eher schlanker. Die Fühlerkeule viel schwächer, als dreigliedrig zu bezeichnen, da das viertletzte Glied zwar ein wenig breiter als lang, aber viel weniger breit als das drittletzte ist. Der Kopf ist sehr schwach punktirt; die Augen ragen ziemlich hervor. Das Halssch. ist schlanker als bei dubia, ähnlich gebaut und hat an der eingedrückten Basis jederseits eine kurze eingedrückte Linie. Die Fld. sind regelmäfsig und fein punktirt-gestreift, am Ende einzeln stumpf zugespitzt.

2 Ex. aus Kamerun von Conradt gesammelt; 1 Ex. aus OldCalabar von Hrn. Donckier.

6. Languria spectabilis: Rufa, capite, elytris, geniculis tarsisque cyanescentibus, antennis nigris, clava lata triarticulata, capile parce subtilissime punctato, thorace subquadrato, parce subtilissime punctato, basi parum impresso, utrinque breviter striolato. Elytris subtiliter punctato-striatis, apice rotundatis, interstitiis striarum haud punctatis. - Long. $9 \frac{1}{2}$ mill. 
Genau von der Grölse des Lang. elateroides Krtz. (D. E. Z. 1895 , p. 202), ganz ähnlich gefärbt, aber weniger gewölbt und durch die sehr feine, weitläufige Punktirung des Kopfes und Halssch. leicht kenntlich. Die Fühler sind schwarz, mit sehr deutlich abgesetzter dreigliedriger Fühlerkeule. Der Kopf ist glänzend blauschwarz, mit einem Eindruck jederseits zwischen den wenig vorstehenden Augen. Das Halssch. ist röthlich, wenig breiter als lang, nach binten leicht verengt, mit scharfen Hinterecken, Basis schwach ausgerandet, an der Basis schwach vertieft, mit einem kurzen aber deutlichen Strichelchen jederseits; die Vorderecken sind schwach herabgebogen, die Oberseite weitläufig, sebr fein punktirt. Das Schildchen ist dreieckig, glatt, mit einem schwachen Stich ins Rötbliche. Die Fld. sind lebhaft blau, fein punktirtgestreift, die $\mathrm{Z}$ wischenräume glatt. Unterseite, Schenkel bis fast zur Spitze und untere Hälfte der Schienen roth.

$1 \mathrm{Ex}$.

7 a. Languria femoralis Fowler (Trans. Ent. Soc. London 1885, p. 386) scheint dieser Art nahe verwandt zu sein, weicht aber von ihr durch meist geringere Gröfse und gelbe Tarsen ab.

7. Languria semirufa Kraatz (D. Ent. Z. 1895, p. 204). Von dieser Art, welche nach einem 7 mill. langen Stück aus Togo beschrieben ist, liegen mir einige Ex. von Lolodorf vor, welche zum Theil nur 6 mill. lang sind. Es wäre daher wohl möglich, dafs semiflava Thoms. (Arch. ent. II, p. 236) auf sie bezogen werden könnte, wenn Thomson's Beschreibung etwas ausführlicher wäre und der Ausdruck "points formant presque des stries" nicht auffallend wäre, da die mir bekannten africanischen Arten nur regelmäfsige Streifen bilden.

8. Languria rufopartita Thomson (Arch. Ent. II, p. 236). Von dieser hübschen Art, die auch bei Togo vorkommt, wurden ein Paar Ex. von Conradt auf Joh. Albrechts-Höhe gesammelt.

Von Thomson's var. a. dieser Art: ,bande transversale sur les élytres effacées" erbielt ich ebenfalls ein Paar Ex., die der semirufa ähnlich sehen, aber sich durch gröfsere Gestalt, den dunkleren Kopf, hellere Fühler und hellrothe Unterseite leicht von derselben unterscheiden lassen; man ist doch zuerst wenig geneigt, sie auf rufopartila Thoms. $z u$ beziehen, da die auffallende helle Binde dieser Art nur durch violette Färbung (auf blauem Grunde) angedeutet ist, sodals ich sie zuerst nicht ohne Weiteres als rufoparlifı erkannte. Aus diesem Grunde scheint es mir angemessen, 
durch den Namen cyanipennis auf die schöne Var. aufmerksam zu machen.

L. rufopartita ist auch bei Kuilu im französischen Congo von 1892 von Mocquerys gesammelt worden (Ex. rom Museum Rothschild in Tring) und in Gabun (Staudinger).

9. Languria gracilior: Elongata, sublinearis, testacea, antennarum articulis 5 ultimis, elytrorum apice femorumque dimidia parte posteriore nigro-piceis, antennarum clava triarticulata, capite fuscescente minus crebre et subtiliter punctato, thorace latitudine longiore, crebre minus subtiliter punctato, utrinque stria impressa, lateribus ante basin subsinuatis, angulis posticis acutiusculis, scutello subrotundato, elytris elongatis, sat fortiter punctato-striatis, apicibus fere rotundatis, sutura haud infuscata, pedibus gracilibus. - Long. $7-7 \frac{1}{2}$ mill.

Durch die Färbung und schlanke, ziemlich flache Gestalt sebr ausgezeichnet. Die Fühler ziemlich schlank, auf der vorderen Hälfte röthlich-gelb, Glied 7 und 8 etwas länger als breit, $9-11$ eine deutlich abgesetzte, aber wenig breite Keule bildend. Kopf meist etwas angedunkelt, Halssch. schlank, in der Mitte bisweilen mit einer schwärzlichen Mittellinie, mäfsig dicht, deutlich punktirt, an der Basis zwischen den vertieften Linien eingedrückt. Die Fld. sind an der Spitze schwärzlich, kräftig punktirt-gestreift, hinten fast abgerundet. Beine schlank, die hintere Hälfte der Schenkel und die Basis der Schieuen schwärzlich.

Einige Stücke von Johann Albrechts-Höhe.

10. Lanquria acuminipennis: Praecedents similis et affinis, sed paullo minor et angustior, magis convexa, rufo-testacea, elytrorum sutura latius tibiisque fuscescentibus, elytris apice acuminatis. - Long. 7 mill.

Mehr röthlich als gelblich-braun, etwas gewölbter als die vorhergehende Art, im Uebrigen ihr recht ähnlich, aber die ganze Naht ziemlich breit geschwärzt (während sie bei der vorbergehenden einfarbig gelblich ist). Die Basis der Schenkel gelblich, aber die ganzen Schienen schwärzlich-braun, jede einzelne Fld. in eine sehr deutliche Spitze vorgezogen, die dadurch noch deutlicher hervortritt, dafs die Fld. an der Basis derselben etwas eingedrückt sind.

2 Ex.

11. Languria macilenta: Minuta, linearis, antennarum femorumque basi pallide testaceis, antennarum clava triarticulata, capile fere laevigato, oculis prominulis, thorace elongato, basin versus 
angustato, convexiusculo, subtilissime punctulato, basi utrinque vix impresso, elytris confertim punctato-striatis, pone humeros, ante medium et post medium leviter oblique impressis. - Long. $3 \frac{1}{2}$ mill.

Eine sehr kleine, schlanke, schwärzliche, glänzende Art mit gelber Basis der Fühler und Schenkel. Die Fühlerkeule ist deutlich abgesetzt, dreigliedrig. Kopf und Halsschild sind glänzend schwarz, letzteres viel länger als breit, ziemlich gewölbt, nach hinten leicht verengt, hinten der Grölse nach vertieft, ein deutlicher Längseindruck jederseits kaum bemerkbar. Skutellum dreieckig. Die Fld. sind verbältnifsmäfsig lang, hinten gemeinschaftlich abgerundet, oben dicht, verhältnifsmälsig stark punktirt-gestreift, drei deutliche Schrägeindrücke auf jeder bemerkbar: einer unterhalb der Schulter, einer etwa in der Mitte und einer hinter derselben.

Nur 1 Ex. Ob die Schrägeindrücke immer vorhanden, kann nur festgestellt werden, wenn mehrere Stücke gefunden worden sind.

12. Von Stenolanguria concolor Kratz (Deutsche Ent. Zeitschr. 1895, p. 207) wurde ein kleines Ex. auf Joh. AlbrechtsHöhe von Conradt gefangen.

Platycladoxena nov. gen.

A genere Cladoxena differt corpore minus nitido et convexo, thorace breviore, angulis posticis haud acuminatis, rectis, elytris punctato-striatis (haud glabris), apicem versus minus angustatis.

Der Name Platycladoxena wird hier in erster Linie für eine Art von Kamerun vorgeschlagen, welche klein, pechbraun, merklich breiter und weniger gewölbt als Paracladoxena von Ceylon ist, die sie an Länge kaum übertrifft; die Fld. sind punktirt-gestreift, nach hinten nur schwach yerengt, an der Spitze gemeinschaftlich zugerundet; die Fld. von Cladoxena Motsch. und Paracladoxena Fowl. sind vor der Spitze eigenthümlich eingedrückt, sodafs die äufserste Spitze schwach schnabelartig vortritt: ein Umstand, der mich veranlassen möchte, beide als Arten einer natürlichen Gattung aufzufassen.

13. Platycladoxena castanea: Brunneo-castanea, nitidula, antennarum clava triarticulata fusca, capite subtilissime punctulato, oculis prominulis, fortius granulatis, thorace subquadrato, leviter convexo, basi latius impresso, sed striolis utrinque vix perspicuis, sculello triangulari, elytris leviter convexis, apicem versus leviter coarctatis, apice rotundatis, confertim subtiliter punctato-striatis, interstitiis subserialim punctulatis. - Long. 4 mill. 
Durch Färbung, geringe Grölse, leichten Glanz unschwer erkennbar, die 3-gliedrige Fühlerkeule sehr deutlich abgesetzt; der Kopf schmäler als das Halssch., wenig dicht und deutlich punktirt, die Augen granulirt. Der Thorax ist ziemlich gewölbt, subquadratisch, die Seiten deutlich gerandet, die Hinterecken rechtwinklig, die Vorderecken etwas herabgebogen, der Hinterrand ziemlich breit abgesetzt, durch eine vertiefte Linie vom Vordertheil abgesetzt, jederseits undeutlich von einer eingedrückten Linie begrenzt; Oberseite weitläufig fein punktulirt, nicht sehr glänzend. Die Fld. sind etwas breiter als die Basis des Halssch., die Schulterecken ein wenig angeschwollen, die Oberseite fein punktirt-gestreift, die Zwischenräume der Streifen mit einer feinen, unregelmälsigen Punktreihe. Beine bräunlich.

Einige Ex. von Kamerun.

Eine der Platycladoxena castanea sehr ähnliche Art besitze ich in 1 Ex. aus Java:

Platycladoxena javanica: Pl. castaneae simillima, brunneocastanea, thorace elytrisque paullo brevioribus et latioribus, apicem versus paullo magis angustatis. - Long. 4 mill.

Eine andere der Pl. castanea ähnliche Art aus Sumatra, im October 90 und März 91 von Modigliani in Pancherang-Pisang gesammelte Art unterscheidet sich leicht von ihr durch das merklich kürzere Halssch.; ich nenne sie

Platycladoxena sumatrana: Brunneo-castanea, antennis (clava triarticulata) pedibusque paullo brevioribus quam in Pl. castanea, thorace longitudine fere duplo latiore, basi simili modo impressa quo in castanea, elytris punctato-striatis. - Long. 3 mill.

Während die beiden ebenso beschriebenen Arten die Basis des Halssch. ähnlich breit vertieft zeigen wie $P l$. castanea, ist dieselbe nur sehr schmal vertieft bei einer kleineren Art von Borneo (Staudinger):

Platycladoxena borneensis: Pl. castanea similis et affinis, sed minor et angustior, brunneo-castanea, antennarum clava triarticulata, concolore, thorace subquadrato, basi minus late impresso, elytris subtiliter punctato-striatis, apicem versus fere laevibus et paullo dilutioribus. - Long. 3 mill. 
Penolanguria Kolbe.

(Käfer von Ostafrica, p. 116.)

Kolbe führt in seinem schönen Werke, die Käfer von Ostafrica, auf S. 116 und 117 vier Languriden-Gattungen auf (Barbaropus Gerh, Languria Latr., Acrolanguria Kolbe und Penolanguria Kolbe), von denen mir die letzte ebenfalls aus Usambara bekannt geworden ist, wenigstens glaube ich Penolanguria minuta Kolbe ${ }^{1}$ ) aus der $1 \frac{1}{4} z$ eiligen Beschreibung zu erkennen. Die Gattungsdiagnose ist zwei Linien lang und lautet: Körper klein, etwas verkürzt, Vorder- und Hinterkörper convex, Augen fein granulirt. Fühlerkeule dreigliedrig, breit; achtes Glied schmal. Wenn Herr Kolbe sich die von ihm brevissime diagnosirte Art etwas näher ansieht, so wird er finden, dafs dieselbe von sä mmtlichen Languriden dadurch abweicht, dafs der Kopf nicht in einer Linie mit dem Halssch. liegt, sondern dafs er so unter demselben verborgen ist, dafs die Stirn mit dem Thorax fast einen rechten Winkel bildet. Wenn man die Abbildungen der Languriiden-Gattungen, welche von Fowler auf Taf. III der Transact. Ent. Soc. 1886 gegeben sind, sieht, wird man die Richtigkeit meines Urtheils bestätigt finden.

Ganz dieselbe Bildung des Kopfes findet sich bei einer kleinen Languria ähnlichen, glänzend schwarzen Art, die mir in $1 \mathrm{Ex}$. von Kamerun und in einem von Old-Calabar vorliegt und die ich deshalb nicht anstehe, auf Penolanguria zu beziehen. Ob Penolanguria überhaupt zu den Languriiden gehört, lasse ich dahingestellt, da das mir vorliegende Material eine nähere Untersuchung nicht gestattet; jedenfalls ist die Gattung nach den von mir oben gemachten Andeutungen zu erkennen; auch werde ich auf die Beschreibung der Art die nothwendige Sorgfait verwenden.

14. Penolanguria nigerrima: Elongata, tota nigerrima, nitida, antennarum clava triarticulata, thorace coleopteris angustiore, convexiusculo, subquadrato, basin versus angustato, angulis posticis obtusis, basi nullo modo impresso, lineis ulrinque nullis, elytris humeris prominulis, subtilissime punctato-striatis. - Long. $4 \frac{1}{2}$ mill.

Einer kleinen Languria ähnlich, aber durch die angegebene Kopfbildung sehr verschieden; auch ist das Halssch. an der Basis leicht der Quere nach rertieft, ohne die gewöhnlichen Längseindrücke der Langurien; die Art ist im Uebrigen durch Kleinheit

1) Zwei der minuta verwandte, aber etwas gröfsere Arten aus Usambara (Weise) liegen mir in je einem Ex. vor. 
und Färbung leicht zu erkennen; das Halssch. ist schmäler als die Fld., die Punktstreifen der letzteren sind kaum zu erkennen.

1 Ex. von Kamerun, 1 Ex. von Donckier.

Penolanguria callosipennis: Penol. minuta major, thorace postice magis impresso, elytris ad scutellum leviter longitudinaliter impressis et callosis, humeris distinctius callosis. - Long. $3 \frac{1}{2}$ mill.

Während mehrere Ex. der Penol. minuta in Färbung und Gestalt ganz gleich sind, zeichnet sich die callosipennis durch ein Paar Längseindrücke innerhalb der Schultern und einen Längseindruck am Schildchen aus, welcher die Schultern als längsbeulig hervortreten und eine Längsbeule jederseits am Schildchen deutlich erkennen läfst.

Penolanguria monacha: Aenescens, thorace posterius angustato, anterius fortius elevato, creberrime subtilissime punctulato, elytris medio gibbulosis, apicem versus angustatis et declivibus. Long. $3 \frac{1}{4}$ mill.

Eine kleine Art von sehr eigenthümlichem Habitus, weil das nach hinten verengte Halssch. nach vorn erbaben ist, sodals es den Vorderrand des herabgebogenen Kopfes überragt; es ist sehr dicht und fein punktulirt, dieses etwas matt erscheinend. Die Fld. sind in der Mitte aufgewölbt, von da nach hinten allmählich verengt und abfallend. Das Halssch. ist länger als breit, hochgewölbt, der Vorderrand kapuzenförmig vorgezogen, sodals die Vorderecken einen stumpfen Winkel bilden; nach hinten ist es ziemlich stark verschmälert, an der Basis leicht eingedrückt und von da ab nach vorn allmählich erhöht. Die gröfste Breite und Höhe liegt in der Mitte der Fld., die fein gestreift sind. Die Fühler haben eine dreigliedrige Keule. 


\section{$2 \mathrm{BHL}$ Biodiversity Heritage Library}

Kraatz, G. 1899. "Ueber die Languriiden-Arten von Kamerun nebst einigen verwandten Formen." Deutsche entomologische Zeitschrift 1899(2), 307-315. https://doi.org/10.1002/mmnd.48018990214.

View This Item Online: https://www.biodiversitylibrary.org/item/103426

DOI: https://doi.org/10.1002/mmnd.48018990214

Permalink: https://www.biodiversitylibrary.org/partpdf/235773

\section{Holding Institution}

Harvard University, Museum of Comparative Zoology, Ernst Mayr Library

\section{Sponsored by}

Harvard University, Museum of Comparative Zoology, Ernst Mayr Library

\section{Copyright \& Reuse}

Copyright Status: Public domain. The BHL considers that this work is no longer under copyright protection.

This document was created from content at the Biodiversity Heritage Library, the world's largest open access digital library for biodiversity literature and archives. Visit BHL at https://www.biodiversitylibrary.org. 